\title{
Comparative Study of Lung Ultrasound and Chest X Ray in Diagnosis of Paediatric Pneumonia - Study of 200 Patients
}

\author{
Mahak Sood ${ }^{1}$, Pinkesh Herma ${ }^{2}$ \\ ${ }^{1}$ Assistant Professor, ${ }^{23}$ rd Year Resident, P.D.U. Government Medical College and Civil Hospital, Rajkot, Gujarat, India \\ Corresponding author: Dr. Pinkesh Herma, 403, Ramvatika Apartment, Marutinagar Street No 3, Airport Road, Rajkot \\ -360001 , India
}

DOI: http://dx.doi.org/10.21276/ijcmsr.2018.3.4.17

How to cite this article: Mahak Sood, Pinkesh Herma. Comparative study of lung ultrasound and chest $\mathrm{X}$ ray in diagnosis of paediatric pneumonia - study of 200 patients. International Journal of Contemporary Medicine Surgery and Radiology. 2018;3(4):D74-D77.

\section{A B S T R A C T}

Introduction: Paediatric pneumonia is the commonest cause of under five mortalities in India. The diagnosis is based on clinical and characteristic radiographic findings. Chest X-Ray is commonly used as initial radiological investigation for the diagnosis of pneumonia in children, which causes radiation exposure. The aim of our study was to replace chest ultrasound as initial and primary modality of pneumonia as an alternative radiological investigation.

Material and Methods: We reviewed 200 cases of paediatric patients with respiratory signs and symptoms and clinically diagnosed as pneumonia who were admitted from September 2017 to February 2018. We exempted cases of serious pneumonia who were admitted at ICU. We perform independent lung ultrasound and chest X Ray of paediatric patients. The final diagnosis was made on the basis of history and clinical findings, laboratory and radiological investigations.

Results: Our results show out of 200 confirmed cases of paediatric pneumonia X ray findings were positive in 192 patients whereas lung ultrasound able to detect 196 patients. 8 patients were diagnosed false negative on $\mathrm{x}$ ray. 4 patients were diagnosed false negative by lung ultrasound.

Conclusion: Lung ultrasound is valuable, valid and non-hazardous alternative diagnostic test for the diagnosis of pneumonia in children and it must be used as initial first line radiological investigation in place of $X$ rays to minimize radiation hazards and improve diagnostic accuracy.

Key words: Pneumonia, Chest x Ray, Lung Ultrasound

\section{INTRODUCTION}

Pneumonia is the most common cause of mortality in children under five years in India. Pneumonia accounts for $19 \%$ of the total number of deaths in children $<5$ years worldwide. Paediatric pneumonia has varying presentations which generally depends on age of child. In a developing country like India, the resources are limited, so paediatric pneumonia remains a diagnostic challenge.

For diagnosis of pneumonia, clinical examination and biochemical investigation alone provides minimal diagnostic accuracy. Radiological examinations like chest X ray and lung ultrasound is necessary to reach the final diagnosis. In current scenario, chest $\mathrm{X}$ ray is the first radiological investigation which is done in paediatric patients with breathlessness and fever. However, it has limitations such as radiation exposure at very young age, nonspecific, normal chest $\mathrm{X}$ ray can never rule out the diagnosis of pneumonia when there is high clinical suspicion.

Recently optional diagnostic tools are being developed for the diagnosis of pneumonia. Lung ultrasound has shown promising results in diagnosis of pneumonia and quantification of its severity. Lung ultrasound is very safe method with no risk of ionizing radiation. Ultrasound is easily available, cheap and non-invasive method of diagnosis. Now -a-days, Portable ultrasound machines are also available for bedside examination. The aim of our study was to replace chest ultrasound as initial and primary modality of pneumonia as an alternative radiological investigation.

\section{MATERIAL AND METHODS}

This Retrospective comparative study was conducted on 200 patients from September 2017 to February 2018 (6 months) in the department of Radio-diagnosis and Imaging, P.D.U. Medical College and Civil hospital, Rajkot, Gujarat over a period of six months from September 2017 to February 2018. Total 200 patients were included in study who underwent chest $\mathrm{X}$ Ray and lung ultrasound. Chest $\mathrm{X}$ ray was done using 100 mAs Allengers MARS 4.2 radiography unit. Lung ultrasound was done using Philips ultrasound system with high frequency linear and low frequency convex probe.

\section{Inclusion criteria}

- Paediatric patients with fever and breathlessness

- Paediatric patients with clinical suspicion of pneumonia

Exclusion criteria

- $\quad$ Age more than 11 years

- Paediatric patients with no respiratory problems 
Methodology

Total 200 patients were analysed by chest $\mathrm{x}$ ray and lung ultrasound. We took detailed history of each patients regarding complaints, course, duration of illness and risk factors. Consent for participation in the study was taken from the patient's parents or guardian. Ethical clearance for the study obtained from ethical committee at P.D.U. Medical college, Rajkot. We did independent chest X ray and lung ultrasound of each patient. Positive findings were recorded, analysed and tabulated. The chest ultrasound was always carried out by the same radiologist with a suitable probe. The probe was placed perpendicular, oblique and parallel to the rib in whole thorax with the patient in the supine position and sitting position to scan the posterior thorax. Diagnosis was made by the sonographic signs of pneumonia are the presence of lung consolidation (hypoechoic area with air sonogram) (Image 1), and presence of pleural effusion, the presence of a sub pleural hypoechoic region with hyperechoic spots of variable size, fluid sonograms, confluent B-lines, superficial fluid alveologram, a vascular tree-shaped pattern. The chest X-ray signs of pneumonia are the presence of heterogeneous radio-opaque area with presence of multiple radiolucent linear tracks which suggest air bronchogram (Image 2) and it is the diagnostic sign of consolidation which is essential to diagnose pneumonia with presence of other related clinical features.

\section{STATISTICAL ANALYSIS}

Statistical analysis was done by comparing positive findings in chest $\mathrm{x}$ ray and lung ultrasound in pediatric patients. Results were analyzed and tabulated in Microsoft office word.

\section{RESULTS}

Total 200 patients with the final diagnosis of pneumonia were analysed. Patients age ranged from 2 months to 11 years. The average hospital stay was around $8 \pm 3$ days without any serious complication and ICU admission.

Our results show that out of 200 confirmed cases of paediatric

\begin{tabular}{|l|c|c|c|}
\hline Chest & $\begin{array}{c}\text { X-Ray } \\
\text { Positive }\end{array}$ & $\begin{array}{c}\text { X-Ray } \\
\text { Negative }\end{array}$ & Total \\
\hline Ultrasound Positive & 156 & 30 & 186 \\
\hline Ultrasound Negative & 10 & 4 & 14 \\
\hline Total & 166 & 44 & 200 \\
\hline Table-1: Radiological investigations in pneumonia positive \\
patients \\
\hline
\end{tabular}

\begin{tabular}{|l|c|c|c|c|}
\hline Age & $\begin{array}{c}\text { Only } \\
\text { ultrasound } \\
\text { positive }\end{array}$ & $\begin{array}{c}\text { Only } \\
\text { chest } \\
\mathbf{X} \text { ray } \\
\text { positive }\end{array}$ & $\begin{array}{c}\text { Both } \\
\text { positive }\end{array}$ & Total \\
\hline <1 Month & 4 & 0 & 28 & 32 \\
\hline 1 Month to 1Year & 5 & 3 & 40 & 48 \\
\hline 1Year to 12Year & 21 & 7 & 88 & 116 \\
\hline Total & 30 & 10 & 156 & 196 \\
\hline Table-2: Age wise distribution of pediatric patients correlated \\
with chest x ray and lung ultrasound \\
\hline
\end{tabular}

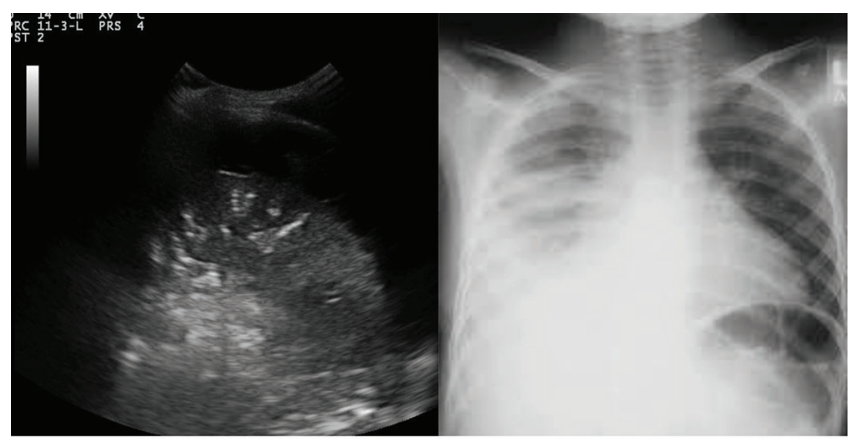

(a)

(b)

Figure-1:(a) shows longitudinal ultrasound scan of the lower zone of right chest in B-mode ultrasound image shows pleural effusion and hypo echoic area with air sonogram suggest consolidation. (b) X-ray chest image shows heterogeneous radio opacity with air bronchograms suggest consolidation with blunting of right cost phrenic angle suggest pleural effusion.

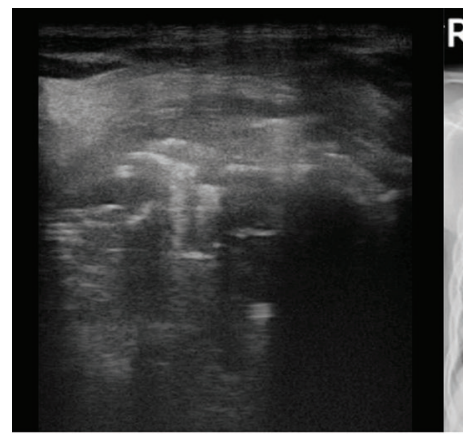

(a)

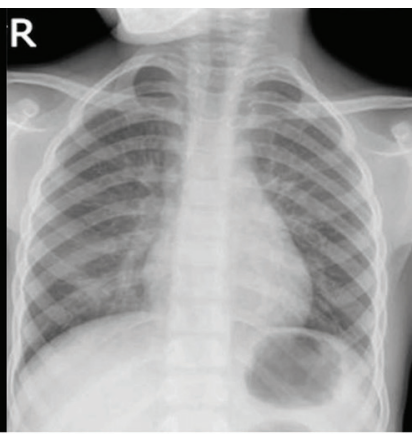

(b)
Figure-2: (a) Longitudinal ultrasound scan of the lower zone of left chest in B-mode ultrasound shows hypo echoic area with air sonogram which suggest presence of consolidation. (b) X-ray chest image shows normal appearance of visualized lung parenchyma on both chest fields

pneumonia, $\mathrm{X}$ ray findings were positive in 166 patients whereas lung ultrasound able to detect 186 patients. 30 patients were diagnosed false negative on $\mathrm{X}$ ray. 10 patients were diagnosed false negative by lung ultrasound. $\mathrm{X}$ ray shows sensitivity and specificity of $75 \%$ and $82 \%$ respectively. Ultrasound shows sensitivity and specificity of $85 \%$ and $92 \%$ respectively. (Table 1)

The presence of air bronchograms was found in 170 of the 200 pneumonias. The presence of air sonograms was found in 186 of the 200 pneumonias. Maximum incidence and detection rate of pneumonia is noted in 2 to 12 year age group. (table 2)

\section{DISCUSSION}

Pneumonia is the one of the important infectious causes of illness in children in India and worldwide. In spite of many treatment options such as various antibiotics and pneumococcal vaccine, pneumonia remains most common cause of morbidity and reason for hospital admission in India. ${ }^{1,2}$

Pneumonia in children is mainly caused by bacteria, which depends on age of presentation. Viruses may also cause 
pneumonia. Presentation of pneumonia vary according to age but main clinical features are cough, breathing difficulty, high grade fever and increased respiratory rate. In chronic cases, it may present as lethargy, failure to thrive and weight loss. ${ }^{3}$

Radiologically, there are two main investigational approaches available. First one is X-ray chest and second one lung ultrasound. X-ray chest is widely available and feasible investigation. But it leads to ionizing radiation exposure and it may also not useful in cases of small area of consolidation such as $<1 \mathrm{~cm}$. The absence of findings on Chest X-ray does not rule out the absence of pneumonia (Figure 2). And in many cases it may be falsely positive which leads to over use of antibiotics. ${ }^{4-10}$

Recently lung ultrasound has shown promising results in detection and staging of pneumonia. Lung ultrasound gives a better idea of the state of the lungs in the analysis of pneumonia because of the thin thoracic walls and large volume of the lungs in children. ${ }^{11}$ By means of lung ultrasound, detection of pleural effusion can be done as low as $15 \mathrm{ml}$ volume whereas $75 \mathrm{ml}$ volume of fluid needed to be evident on chest $\mathrm{x}$ ray. Lung ultrasound also provides useful information about nature of pleural effusion. Lung ultrasound provides an idea about quantity, nature, septations, and content of effusion. With help of ultrasound it is easy to detect complication like empyema earlier than before. ${ }^{12}$

Ultrasound can be conducted as a bedside investigation which makes results instantly available. Only limitation for lung ultrasound is expertise of operator. Chest $\mathrm{x}$ ray is relatively easy to perform while detection and staging of pneumonia by lung ultrasound needs experts hand and years of practice. ${ }^{13,14}$

Our study shows that lung ultrasound is the best initial investigation in paediatric patients for detecting and staging the severity of pneumonia. As compared to conventional approach where chest $\mathrm{x}$ ray is recommended as first line investigation, lung ultrasound is more specific and sensitive not only for early diagnosis but also enables us to detect complication early without risk of ionizing radiation. . $^{15,16}$

Reissig et al suggest community acquired pneumonia may be diagnosed and followed up by lung sonography (LUS), a technique that shows excellent sensitivity and specificity that is at least comparable with that of chest X-ray in two planes. LUS may be performed with any abdomen-sonography device. Therefore, LUS is a readily available diagnostic tool that does not involve radiation exposure and has wide applications especially in situations where X-ray is not available and/or not applicable. An X-ray or CT of the chest should be performed in cases of negative lung sonography and if other differential diagnoses or complications are suspected. ${ }^{16}$

Copetti et al concluded that Lung ultrasound is a simple and reliable tool that can be used by the clinician in the case of suspected pneumonia. It is as reliable as chest $\mathrm{x}$ ray, can be easily repeated at the patient's bedside and carries no risk of irradiation. $^{2}$

Iuri et al shows out of 52 selected medical records community acquired pneumonia diagnosis was confirmed in 29 (55.7\%). Chest $\mathrm{x}$ ray was positive in 25 cases, whereas lung ultrasound detected pneumonia in 28 cases. Four patients with negative Chest $\mathrm{x}$ ray were positive in ultrasound findings. Instead, one patient with negative lung ultrasound was positive in radiographic findings. The lung ultrasound sensitivity was 96.5\% (95\% CI [82.2\%-99.9\%]), specificity of 95.6\% (95\% CI [78.0\%-99.9\%]), positive likelihood ratio of $22.2(95 \%$ CI [3.2-151.2]), and negative likelihood ratio of 0.04 (95\% CI [0.01-0.25]) for diagnosing pneumonia. Study proved lung ultrasound can be considered as a valid alternative diagnostic tool of community acquired pneumonia in children and its use must be promoted as a first approach in case of community acquired pneumonia. ${ }^{12}$

\section{CONCLUSION}

Lung ultrasound shows very good reliability and accuracy in the diagnosis of pneumonia as the first line investigation. So we recommended the lung ultrasound as the primary radiological diagnostic tool even before chest $\mathrm{x}$ ray for paediatric pneumonia.

\section{REFERENCES}

1. Caiulo VA, Gargani L, Caiulo S, Fisicaro A,Moramarco F, Latini G, Picano E,Mele G. Lung ultrasound characteristics of community-acquired pneumonia in hospitalized children. Pediatric Pulmonology 2013; 48(1):280-287.

2. Copetti R, Cattarossi L. Ultrasound diagnosis of pneumonia in children. Radiologia Medica 2008;113 (3):190-198.

3. Iuri D, De Candia A, Bazzocchi M. Evaluation of the lung in children with suspected pneumonia: usefulness of ultrasonography. Radiologia Medica 2009; 114 (5):321-330.

4. Miller RW. Special susceptibility of the child to certain radiation-induced cancers. Environmental Health Perspectives 1995; 103 (6):41-44.

5. Esposito S, Papa SS, Borzani I, et al. Performance of lung ultrasonography in children with communityacquired pneumonia. Ital J Pediatr. 2014;40 (2):37.

6. Copetti R, Cattarossi L. Ultrasound diagnosis of pneumonia in children. Radiol Med. 2008;113(2):190198.

7. Shah VP, Tunik MG, Tsung JW. Prospective evaluation of point-of-care ultrasonography for the diagnosis of pneumonia in children and young adults. JAMA Pediatr. 2013;167 (4):119-125.

8. Pereda MA, Chavez MA, Hooper-Miele CC, et al.Lung ultrasound for the diagnosis of pneumonia in children: a meta-analysis. Pediatrics. 2015;135(4):714-722.

9. World Health Organization. Pneumonia. Fact Sheet No 331. Geneva, Switzerland. Available from: www. who.int/mediacentre/factsheets/fs331/en/. Accessed March 22, 2015.

10. Esposito S, Papa SS, Borzani I, et al. Performance of lung ultrasonography in children with communityacquired pneumonia. Ital J Pediatr. 2014;40 (1):37.

11. Shah VP, Tunik MG, Tsung JW. Prospective evaluation of point-of-care ultrasonography for the diagnosis of pneumonia in children and young adults. JAMA Pediatr. 2013;167(2): 119-125.

12. Iuri D, De Candia A, Bazzocchi M. Evaluation of 
the lung in children with suspected pneumonia: usefulness of ultrasonography. Radiol Med (Torino). 2009;114(2):321-330.

13. Reali F, Sferrazza Papa GF, Carlucci P, et al. Can lung ultrasound replace chest radiography for the diagnosis of pneumonia in hospitalized children? Respiration. 2014;88(2):112-115.

14. Caiulo VA, Gargani L, Caiulo $S$, et al. Lung ultrasound characteristics of community-acquired pneumonia in hospitalized children. Pediatr Pulmonol. 2013;48(3):280-287.

15. Lichtenstein DA, Mauriat P. Lung ultrasound in the critically ill neonate. Curr Pediatr Rev. 2012;8(3):217223.

16. Reissig A, Gramegna A, Aliberti S. The role of lung ultrasound in the diagnosis and follow-up of community-acquired pneumonia. Eur J Intern Med. 2012;23(5): 391-397.

Source of Support: Nil; Conflict of Interest: None

Submitted: 28-08-2018; Accepted: 14-11-2018; Published online: 26-11-2018 